\title{
Perceptual specificity in visual object priming: functional magnetic resonance imaging evidence for a laterality difference in fusiform cortex
}

\author{
W. Koutstaal ${ }^{\mathrm{a}, *}$, A.D. Wagner ${ }^{\mathrm{b}, \mathrm{c}}$, M. Rotte $^{\mathrm{c}}$, A. Maril ${ }^{\mathrm{a}}$, R.L. Buckner ${ }^{\mathrm{c}, \mathrm{d}}$, \\ D.L. Schacter ${ }^{\mathrm{a}}$ \\ a Department of Psychology, Harvard University, William James Hall, 33 Kirkland Street, Cambridge, MA 02138, USA \\ ${ }^{\mathrm{b}}$ Department of Brain and Cognitive Sciences, MIT, Cambridge, MA 02139, USA \\ c MGH-NMR Center, Harvard University Medical School, Charlestown, MA 02129, USA \\ d Departments of Psychology, Radiology, and Anatomy \& Neurobiology, Washington University, St Louis, MO 63130, USA
}

Received 04 October 1999; received in revised form 20 May 2000; accepted 20 June 2000

\begin{abstract}
Seeing an object on one occasion may facilitate or prime processing of the same object if it is later again encountered. Such priming may also be found - but at a reduced level — for different but perceptually similar objects that are alternative exemplars or 'tokens' of the initially presented object. We explored the neural correlates of this perceptual specificity using event-related functional magnetic resonance imaging (fMRI) procedures, contrasting neural activity when participants made object classification decisions (size judgments) regarding previously presented objects (repeated same), alternative exemplars of previously presented objects (repeated different), or entirely new objects (novel). Many frontal regions (including bilateral frontal operculum, bilateral posterior inferior frontal/precentral, left anterior inferior frontal, and superior frontal cortices) and multiple late visual and posterior regions (including middle occipital, fusiform, fusiform-parahippocampal, precuneus, and posterior cingulate, all bilaterally), demonstrated reduced neural activity for repeated compared to novel objects. Greater repetition-induced reductions were observed for same than for different exemplars in several of these regions (bilateral posterior inferior frontal, right precuneus, bilateral middle occipital, bilateral fusiform, bilateral parahippocampal and bilateral superior parietal). Additionally, right fusiform (occipitotemporal) cortex showed significantly less priming for different versus same exemplars than did left fusiform. These findings converge with behavioral evidence from divided visual field studies and with neuropsychological evidence underscoring the key role of right occipitotemporal cortex in processing specific visual form information; possible differences in the representational-functional role of left fusiform are discussed. (c) 2000 Elsevier Science Ltd. All rights reserved.
\end{abstract}

Keywords: Repetition priming; Object recognition; Basal temporal language area

\section{Introduction}

Human processing of visual forms is characterized by two broadly complementary abilities. On the one hand, we can readily notice what is the same across differing perceptual inputs. Thus, 'a cup, is a cup, is a cup' regardless of whether the object before us is a coffee mug, a tea cup, or a measuring cup. On the other hand, we can also notice (sometimes with surprising acuity) what it is that distinguishes one particular object from others

* Corresponding author. Tel.: + 44-118-931-6668.

E-mail address: w.koutstaal@reading.ac.uk (W. Koutstaal). that are, in many respects, very similar. We can differentiate our own cup from someone else's, our own car from among many others, and (if possessed of the appropriate training and interest) a particular type of bird, fish, or plant from among numerous other birds, fish, or plants. The existence of these two, broadly different, forms of perceptual and cognitive processing raises several questions: What cognitive and neural processes support our ability to extract and use comparatively abstract versus highly specific information about visual forms $[29,52,49]$ ? Are different regions of the brain responsible for processing specific visual form information as opposed to more abstract types of information [23,32,37]? 
One approach to these questions — particularly with regard to understanding how differing individual exemplars of an object, such as two different umbrellas, may be processed - is to examine priming effects with an indirect or implicit measure of memory for previously encountered objects [19,54]. In a priming experiment, individuals perform a perceptual or semantic task that does not require explicit recollection of their initial experience with an object. Speed or accuracy of responding to repeated objects, compared to new objects, is used to draw inferences about the cognitive processes engaged during the initial experience, and the nature of the information retained in memory. Results from priming experiments suggest that specific visual information is sometimes preserved. Studies of visual object priming, using such tasks as naming and perceptual identification, have found less repetition priming when a test object is a different exemplar or token of a previously presented object (e.g. a different umbrella from that originally presented) than when a test object is the same exemplar $[2-4,12,68]$. Other forms of perceptual changes in words and objects have also been observed to reduce priming [e.g. [24,59]; for review see [50]].

Several studies have further suggested that sensitivity to alterations in the precise perceptual form of a stimulus may be influenced by the laterality of stimulus presentation. Using a 'divided visual field' paradigm in which stimuli were initially presented centrally but then, at test, were briefly presented either to the left or right visual field, Marsolek and colleagues [36,37,39] reported greater decrements in priming when perceptually altered stimuli were presented directly to the right than to the left cerebral hemisphere. This outcome, found for words, pseudo-words, word-like forms, and line drawings of common objects [35], implies that the right cerebral hemisphere may retain more specific visual form information than the left, which may store relatively more abstract forms or lexical-semantic representations. Neuropsychological evidence from patients with damage to occipitotemporal cortex leading to an impaired ability to recognize faces (prosopagnosia) is also consistent with the notion that areas of right occipitotemporal cortex may be particularly sensitive to alterations in exemplar form. In a number of cases, damage to these regions also impaired within-category or item-specific visual object recognition, with such item-specific impairments particularly (although not exclusively) found for lesions in the right hemisphere $[7,17,18,23,69]$. The work of Gabrieli and colleagues $[21,62]$, demonstrating impaired visual form specific priming for words in a patient with right occipital resection (performed as treatment for intractable epilepsy, and including Brodmann areas 17 and 18 and a part of area 19) also points to the possible role of the right hemisphere in contributing to visual form specific priming. In particular, Vaidya et al. [62] found that patient M.S. showed no 'font-specific' priming in a word-stem completion task (although showing normal levels of priming for words shown in a different font at study versus test, unlike the controls, M.S. showed no benefit from the reinstatement of the same font relative to a different font).

Recently, several studies have examined the neural correlates of repetition priming in cognitively intact humans using positron emission tomography (PET) and functional magnetic resonance imaging (fMRI). These studies have shown that the behavioral priming that results from repeated exposure to a stimulus also is associated with indications of enhanced 'neural efficiency': some of the brain regions initially involved in the processing of a stimulus show reduced activity during repeated processing of the stimulus. Reductions in neural activity for repeated compared to novel stimuli have been observed in several regions of left frontal cortex that are believed to be involved in accessing or evaluating semantic or phonological representations (Brodmann areas 47, 45, 44) [10,16,20,44]. Similar reductions have been observed in several posterior cortical regions involved in perceptual or form recognition, including occipitotemporal and occipital cortices $[[8,9,40,55,58]$; for review see [54,70]].

The aim of the present study was to examine the neuroanatomical correlates of priming in a visual object classification task under conditions where the object either remained the same across repetitions (e.g. 'umbrella A' on all presentations) or was a different exemplar ('umbrella $A$ ' on the initial presentations, but 'umbrella B' on the critical test trial). Although previous neuroimaging studies of priming have used various types of stimuli, including words [e.g. [16]] and objects $[9,40,64]$, in almost all prior studies ${ }^{1}$ the repeated stimulus was the same as, or a part of $[5,55,58]$, the initially exposed stimulus. Thus, these studies do not address the important question of whether reductions in behavioral priming for different exemplars are accompanied by similar modulations in neural correlates of priming, nor do they address whether left and right occipitotemporal regions are differentially sensitive to such changes in the match between the visual form information processed during initial and repeated presentations.

Using whole-brain event-related fMRI procedures that allow examination of regional brain activity associated with randomly and rapidly intermixed trial types [14], we compared neural measures of priming for same

\footnotetext{
${ }^{1}$ One exception is Buckner et al. [8] who examined the consequences of changing the letter case of visually presented word-stems across repeated exposures. Their results, however, could not be used to make relative comparisons between same and different visual formats because a same vs. different case manipulation was not performed within the same study.
} 
and different object exemplars. In addition, we focused on how alterations in the perceptual form of repeated objects might modulate priming-related reductions in neural activity in right compared to left occipitotemporal regions. We selected as a particular region of interest an area of fusiform cortex (BA 37/19) that demonstrated robust repetition-related decreases in activity in an earlier object priming study where only identical objects were repeated [9], and that also showed increased activation under conditions where participants made subordinate category judgments regarding an object (is this a sparrow?) relative to superordinate judgments (is this a bird?) [22,23]. The latter effect may possibly reflect the additional perceptual processing required to arrive at a more specific (withincategory) decision. If right fusiform cortex processes comparatively more specific visual form information about previously encountered objects, then this region might show less pronounced reductions in neural activity for different exemplars than does left fusiform cortex.

\section{Method}

\subsection{Participants}

Eighteen individuals (eight female) took part in the experiment. All were right-handed, native speakers of English (ages 18-32), with normal or corrected-to-normal vision, and received $\$ 50$ for their participation.

\subsection{Stimuli}

The stimuli were 216 picture pairs (colored line drawings), representing simple objects, obtained from CDROM clip-art collections (e.g. Corel Mega Gallery, Corel Corporation, 1997). The items in each pair represented two exemplars or 'tokens' of a given object (e.g. two umbrellas) and were selected to be as different from one another as possible in shape, color, surface pattern, and material. Orientation also varied (no objects were shown in unusual views), but exemplars were chosen primarily on the basis of features of the object itself. Although, in a few instances, some of the participants may have treated the objects within a pair as differing across levels of categorization (e.g. snake and constrictor), rather than as two different exemplars at the same categorization level (both snakes), the objects in a pair were selected as likely to be designated with the same name by the majority of participants — while still attempting to maximize perceptual differences within each pair. Consistent with this, a normative behavioral study conducted with 12 participants (eight female, ages 18-24) from the same population as those who took part in the imaging study demonstrated that, on average, within each participant, approx. $91 \%$ of the objects within the object pairs were given either exactly the same name $($ mean $=81 \%$, range $=76-86 \%)$, or a minor variant of the name $($ mean $=10 \%$, range $=6-$ $12 \%$; e.g. flower vs. flowers; TV set vs. television set; pail vs. bucket; wheel vs. tire; errors, involving naming the object something that was entirely unrelated, or not naming the object within five seconds, occurred on $1 \%$ of the trials). Across participants, the objects were systematically counterbalanced across conditions (novel, repeated same, repeated different). ${ }^{2}$

\subsection{Behavioral procedure}

The experimental session consisted of four alternating 'study-test' cycles, preceded by a brief study-test practice demonstration. The purpose of the study-test practice demonstration was twofold: first, to ensure that participants understood and could successfully complete the task, and second, to pre-familiarize them with the likely occurrence of, and the nature of, the repeated items that would be presented during the experiment, including repeated same, repeated different, and novel exemplars, thereby minimizing within-session changes in how participants might perform the task.

A (non-scanned) study phase occurred immediately prior to each (scanned) test run. In each study phase, a new subset of 36 objects was presented four times, in a continuous randomly intermixed sequence, using a new random order each time. (The objects were presented repeatedly rather than only once because prior research [e.g. [9]] demonstrated increased behavioral priming for repeated same items that were presented multiple times and we wanted to maximize the likelihood of detecting any neural differences in priming for the repeated same and repeated different items.)

\footnotetext{
${ }^{2}$ For counterbalancing purposes, the set of 216 object pairs was divided into four sets equated for content type (e.g. animals, furniture, etc.); these sets were further divided into three subsets. The sets were used to counterbalance stimuli across the four runs of the experiment and subsets were used to counterbalance conditions within runs (novel, repeated same, repeated different). Additionally, within each object pair, the objects were pseudo-randomly assigned to either an odd or an even stimulus number. For the different exemplar condition, the items shown at study were always the exemplars with odd numbers; at test, and for all conditions, only even-numbered exemplars were tested. In this way, all participants were tested with the identical set of 216 objects but the prior 'exposure history' for the objects was systematically counterbalanced (i.e. earlier preceded by the odd-numbered pair-member for different items, by the even-numbered pair-member for the same items, and by no prior exposure for novel items). Because the stimuli presented during scanning were held constant for all participants - only exposure history varied - any differences between the three key object presentation conditions (repeated same, repeated different, and new) cannot be attributed to irrelevant or uncontrolled perceptual differences in the stimuli within a pair.
} 


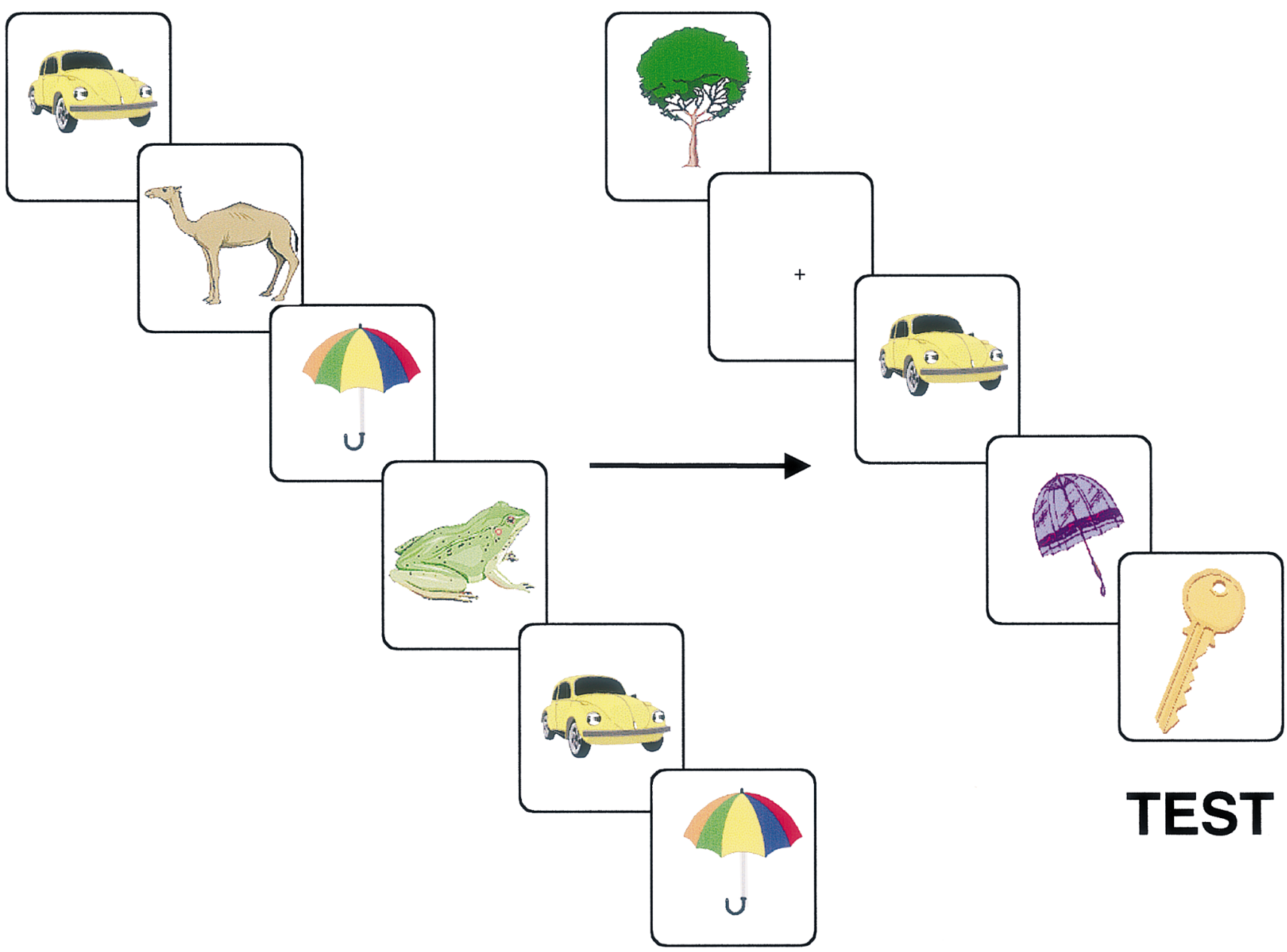

\section{STUDY}

Fig. 1. Examples of the colored objects used as stimuli in the experiment. Items during study were shown at a rate of one stimulus every two seconds and were presented four times in a continuous, randomly intermixed, list. Trial types at test included novel objects (items never previously presented in the experiment), repeated same (items identical to those presented in the immediately prior study phase), repeated different (items that were alternative exemplars or 'tokens' of items presented in the immediately prior study phase) and visual fixation.

Stimuli were presented once every two seconds $(0.5 \mathrm{~s}$ stimulus duration; fixation cross-hair displayed between stimuli). Participants decided if the referent of each object was or was not larger than a 13 in. square box. To provide a concrete frame of reference for this judgment, participants were shown an actual 13 in. square box during the task instructions. They were instructed to respond quickly and accurately and to maintain fixation throughout the task.

In the test phase, participants performed the same object classification task, but now during scanning. Items were presented in randomly intermixed (nonblocked) order, with test runs comprised of four trial types: novel (objects not previously presented), repeated same (identical to objects repeatedly presented during the immediately preceding study phase), repeated different ('alternative' exemplars of objects repeatedly pre- sented during the immediately preceding study phase), and fixation (a centrally displayed visual cross-hair). All objects were presented once only. Fig. 1 presents a schematic diagram of the procedure. ${ }^{3}$

\footnotetext{
${ }^{3}$ For each test, pseudo-random orderings of the four trial types were created for individual participants, ensuring that each trial type occurred equally often and that each trial type was approximately equally likely to be preceded, on the two immediately prior trials, by all combinations of the other trial types ([11]; see [9] for additional details). Each of these initial trial orders was used to systematically counterbalance the ordering of the trial types for a given participant such that, across the four runs, any one trial position (e.g. the first item of each run) represented each of the four conditions (novel, repeated same, repeated different, and fixation). In this manner, the average 'trial history' of each trial type was equated, thus allowing for cancellation of the overlap ('cross-talk') of the hemodynamic response across trials (see [14]). Each of the first 12 participants received a unique counterbalancing; thereafter, a subset of six of these 12 sets of lists was used for the remaining participants.
} 


\section{4. fMRI imaging procedure}

Imaging was performed with a $3.0 \mathrm{~T}$ General Electric MRI scanner equipped with an echo planar imaging upgrade (Advanced NMR Systems). Visual images were back-projected to participants from an Apple PowerMacintosh computer; participants viewed the images through a mirror on the head coil. To minimize artifacts arising from head motion, foam cushioning was placed snugly around the side and back of the participant's head. Participants indicated their responses to the object classification task with their left (non-dominant) hand using a magnet-compatible key press.

Conventional structural images that provided detailed anatomic information were first acquired (high resolution rf-spoiled GRASS sequence, 60 slice sagittal, $2.8 \mathrm{~mm}$ thickness), followed by functional images sensitive to blood oxygenation level-dependent contrast (echo planar $T_{2}^{*}$-weighted gradient echo sequence, $\mathrm{TR}=2 \mathrm{~s}, \mathrm{TE}=30 \mathrm{~ms}$, flip $=90^{\circ}$ ). Each functional run consisted of 88 sequential whole-brain acquisitions (16 axial slices aligned to the plane intersecting the anterior and posterior commissures, $3.125 \mathrm{~mm}$ in-plane resolution, $7 \mathrm{~mm}$ thickness, skip $1 \mathrm{~mm}$ between slices). ${ }^{4}$

The procedures for selective averaging and statistical map generation are detailed elsewhere [9,14]. Briefly, data from individual fMRI runs were first normalized to correct for global between-run signal intensity changes and temporal drift. The normalized data were then selectively averaged in relation to the beginning of each trial type. There were five trial types: novel, repeated same, repeated different, fixation, and 'other' (trials where no response was given, or the response occurred in $<300 \mathrm{~ms}) .{ }^{5}$ After all trials were selectively averaged for each subject, the mean and variance images were transformed into stereotaxic atlas space (interpolated to $3.125 \mathrm{~mm}$ isotropic voxels) allowing for across subject averaging $[60,61]$. Thereafter, statistical activation maps were constructed based on the differences between trial types using a $t$-statistic [14].

Clusters of five or more voxels $\left(152.6 \mathrm{~mm}^{3}\right)$ exceeding a statistical threshold of $p<0.001$ were considered sig-

\footnotetext{
${ }^{4}$ To provide a stable task baseline, each functional run began and ended with a $16 \mathrm{~s}$ period of visual fixation. Also, at the beginning of each run, four additional whole-brain images were acquired and discarded to allow longitudinal magnetization to reach equilibrium.

${ }^{5}$ During fMRI analysis, we excluded items where participants gave no response, or provided a response in $<300 \mathrm{~ms}$. Approximately $1 \%$ of the trials were excluded for these reasons. Although the exclusion of these items slightly altered the counterbalancing for individual subjects, the number of such trials was few, and examination of the time-courses (see Fig. 4) suggests that the counterbalancing was successful (note the close equivalence of the activation levels for each of the three conditions at the onset of the trial).
}

nificant foci of activation. These criteria have been found to result in the identification of few false positives in control data sets $[9,66]$. An automated algorithm was used to identify significant peaks of activation; when significant peaks occurred within 10 $\mathrm{mm}$ of one another, the most significant peak was retained. For a subset of the peak activations that were obtained in an unbiased comparison of novel trials vs. all repeated trials, additional region-of-interest (ROI) analyses were conducted. For these ROIs, analyses of variance (ANOVAs) - treating subjects as a random factor - were performed for each ROI separately, treating object type (novel, repeated same, repeated different) and time-point (time-point 1 through timepoint 8 , corresponding to the eight images acquired during the $0-16 \mathrm{~s}$ peri-stimulus window) as repeated measures factors; the dependent variable was the percent MRI signal change from the average signal across all trials, less fixation. Where these initial analyses showed a significant object type $\times$ time-point interaction, further analyses were conducted on the peak (highest amplitude) response, defined using the mean of all three object types. These included, first, one-way ANOVAs treating object type as a repeated measures factor, and second (where these one-way analyses showed significant differences), focused pairwise comparisons, contrasting each of the conditions against each other. Finally, a ROI analysis was performed to test for possible laterality differences in repetition priming in right and left occipitotemporal (fusiform) cortex for repeated same vs. repeated different exemplars.

\section{Results}

\subsection{Behavioral results}

Participants showed a high level of accuracy on the object classification task both during the initial non-

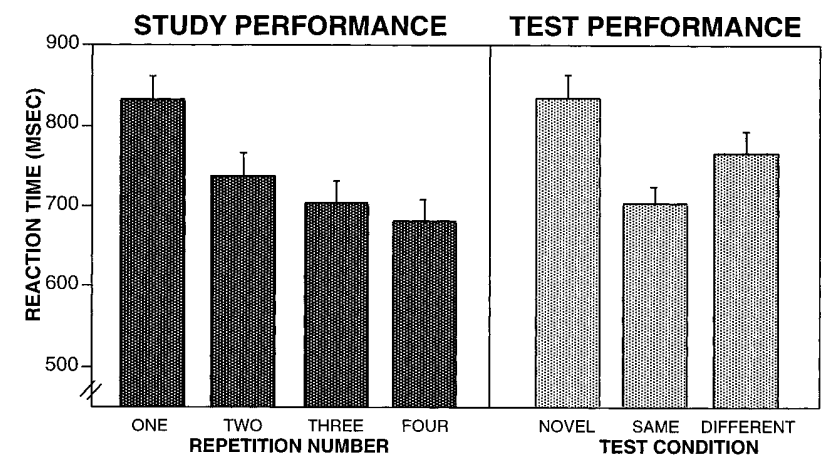

Fig. 2. Mean response latencies for the object classification task across the four repeated presentations of objects during the study phase (left panel) and for novel, repeated same, and repeated different objects in the test phase (right panel). Error bars indicate standard errors of the mean. 
Table 1

Regions demonstrating greater activation for novel than repeated objects (combining across same and different objects) ${ }^{\mathrm{a}}$

\begin{tabular}{|c|c|c|c|c|c|}
\hline \multirow[t]{2}{*}{ Region } & \multicolumn{3}{|c|}{ Talairach coordinates } & \multirow[t]{2}{*}{ Significance $-\log (p)$} & \multirow[t]{2}{*}{ BA } \\
\hline & $x$ & $y$ & $z$ & & \\
\hline $\mathrm{L}$ frontal operculum & -31 & 22 & 9 & 22.60 & 45,47 \\
\hline $\mathrm{L}$ anterior inferior frontal & -31 & 34 & -3 & 22.47 & 47,45 \\
\hline L parahippocampal & -21 & -37 & -6 & 21.34 & 36 \\
\hline Superior frontal & 6 & 16 & 46 & 17.90 & 6,8 \\
\hline $\mathrm{R}$ fusiform & 46 & -58 & -6 & 17.45 & 37,19 \\
\hline L fusiform/parahippocampal & -31 & -43 & -15 & 15.21 & 37,36 \\
\hline $\mathrm{L}$ precuneus & -25 & -68 & 31 & 15.10 & 31,19 \\
\hline $\mathrm{R}$ frontal operculum & 34 & 19 & 6 & 14.87 & 45,47 \\
\hline $\mathrm{R}$ middle occipital/middle temporal & 46 & -74 & 18 & 14.24 & 19,39 \\
\hline $\mathrm{R}$ middle occipital & 34 & -74 & 15 & 12.59 & 19 \\
\hline $\mathrm{L}$ posterior cingulate & -9 & -52 & 15 & 11.31 & 30,23 \\
\hline $\mathrm{R}$ superior parietal & 25 & -52 & 46 & 10.69 & 7 \\
\hline $\mathrm{R}$ posterior inferior frontal & 40 & 9 & 31 & 10.13 & 44 \\
\hline $\mathrm{R}$ middle occipital/fusiform & 34 & -68 & -9 & 9.80 & 19 \\
\hline $\mathrm{R}$ posterior cingulate & 12 & -49 & 6 & 9.66 & 29,30 \\
\hline $\mathrm{R}$ fusiform & 18 & -68 & -9 & 9.64 & 19 \\
\hline $\mathrm{R}$ cerebellum & 28 & -62 & -15 & 9.43 & - \\
\hline $\mathrm{R}$ posterior inferior frontal & 46 & 9 & 21 & 9.10 & 44 \\
\hline $\mathrm{R}$ precuneus & 21 & -55 & 12 & 8.78 & 31,23 \\
\hline $\mathrm{R}$ precuneus & 28 & -68 & 28 & 8.29 & 31,19 \\
\hline $\mathrm{L}$ middle temporal/inferior parietal & -43 & -68 & 18 & 8.22 & 39 \\
\hline
\end{tabular}

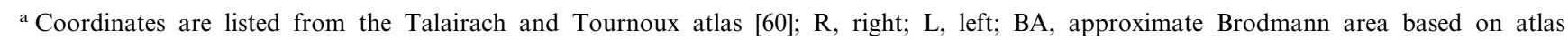
coordinates.

scanned study phase $(86.2 \%)$ and during the fMRI test phase $($ novel $=83.3 \%$, repeated same $=87.6 \%$, repeated different $=88.2 \%$ ). Classification judgments for novel items were significantly less accurate than for repeated same, $F(1,17)=10.44, M S E=0.002, P=0.005$, or repeated different, $F(1,17)=10.39, M S E=0.002, P=$ 0.005 , exemplars.

Classification response times during the initial study phase decreased across the four repeated presentations of an object (Fig. 2), showing substantial response facilitation or priming, $F(3,51)=83.72, M S E=959.14$, $P<0.0001$. This priming effect carried over into the scanned test phase, with both repeated same (mean $\mathrm{RT}=701 \mathrm{~ms})$ and repeated different $(769 \mathrm{~ms})$ items classified more quickly than novel items $(835 \mathrm{~ms}), F(1$, $17)=98.52, M S E=1643.72, P<0.0001$ and $F(1,17)=$ 48.33, $M S E=823.69, P<0.0001$, respectively. Critically, priming was also influenced by the degree of perceptual similarity of the repeated items: classification responses were reliably faster for repeated same than for repeated different exemplars, $F(1,17)=46.11$, $M S E=892.90, P<0.0001$. Additionally, examination of the pattern of behavioral priming separately for each of the four study-test runs indicated that participants showed a similar pattern of priming performance across the entire scanning session, with each of the four runs yielding both significant priming and a significant specificity effect (for repeated different $>$ repeated same, smallest $F(1,17)=22.66, \quad M S E=936.51, P<0.0002)$, and no overall effect of run on the magnitude of the specificity effect, $F(3,51)<1.9$. These outcomes suggest that participants continued to perform the task in a similar manner throughout the scanning session.

\subsection{Imaging results}

\subsubsection{Novel $>$ repeated comparisons}

We first considered brain regions that showed greater activity for novel items than for All repeated items (combining across repeated same and repeated different). As shown in Table 1 and Fig. 3, greater activation for novel than all repeated items was observed in multiple bilateral late visual and posterior regions, including middle occipital, fusiform, fusiform/ 
NOVEL > ALL REPEATED

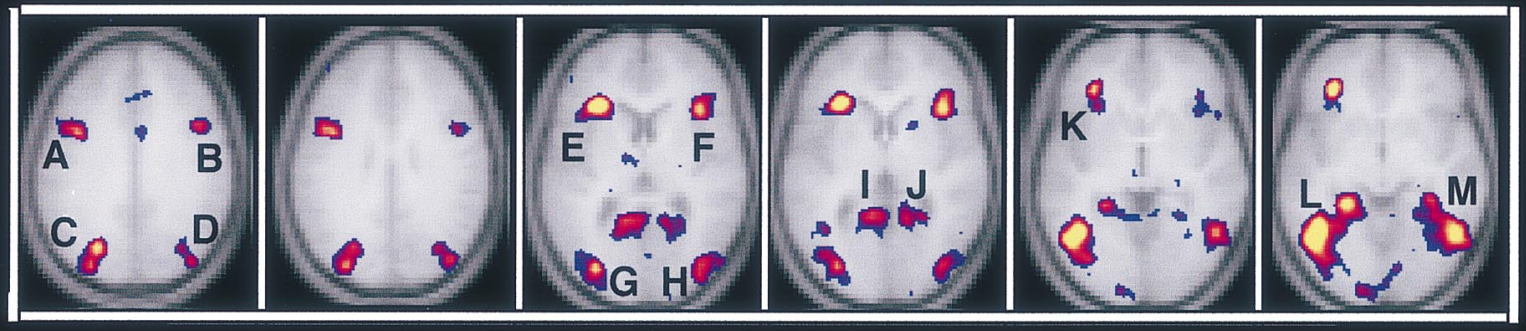

NOVEL $>$ REPEATED SAME

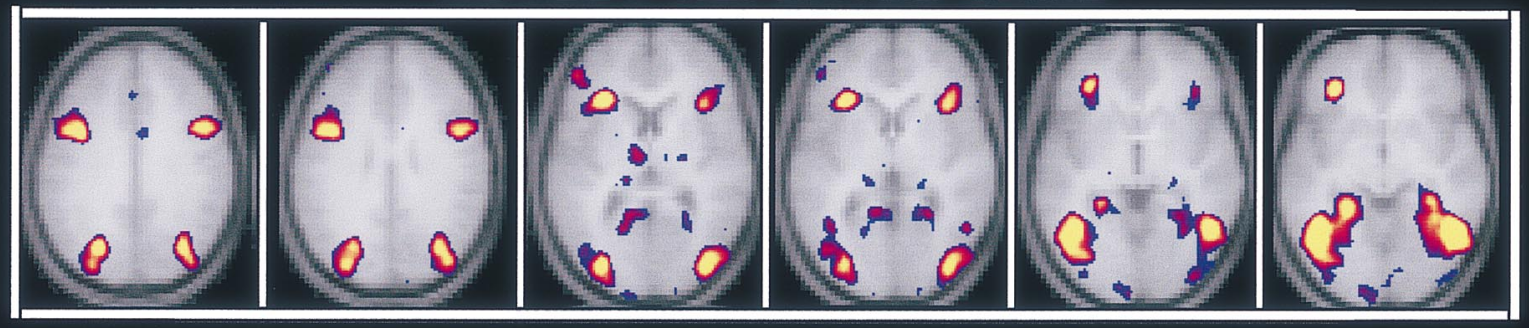

NOVEL $>$ REPEATED DIFFERENT

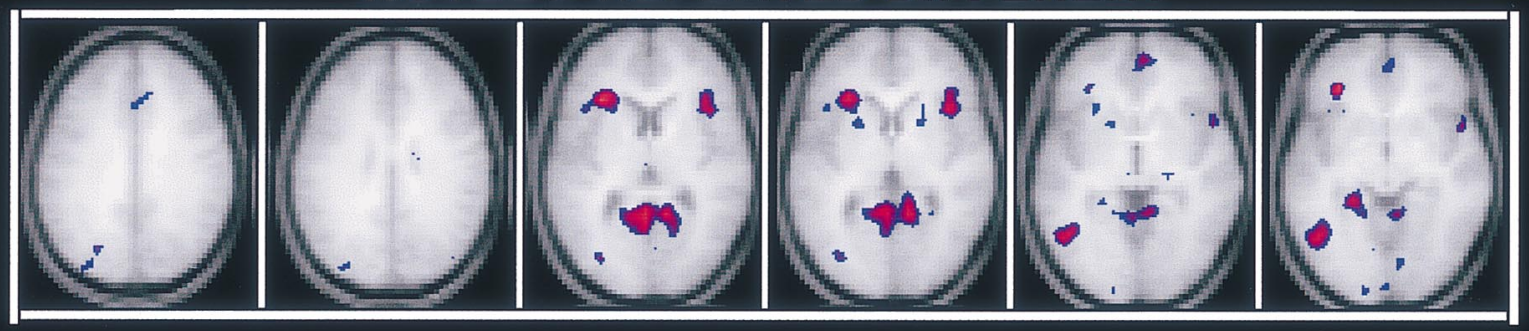

REPEATED DIFFERENT > REPEATED SAME

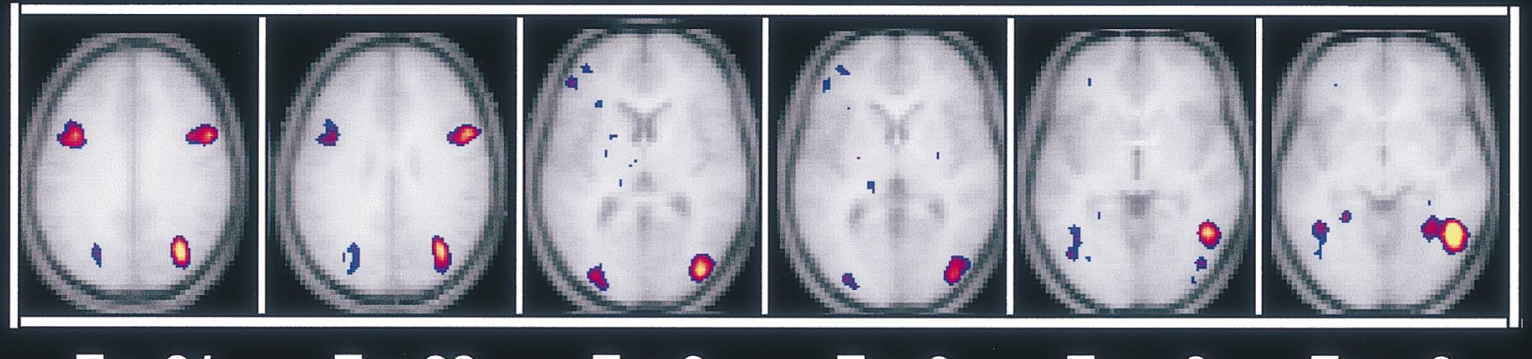

$$
\begin{array}{llllll}
Z=31 & Z=28 & Z=9 & Z=6 & Z=-3 & Z=-6
\end{array}
$$

$$
P<.01
$$

$$
P<10^{-6}
$$

Fig. 3.

parahippocampal, precuneus, and posterior cingulate. Several frontal regions were also more active for novel than all repeated items, including bilateral frontal oper- culum, bilateral posterior inferior frontal/precentral, left anterior inferior frontal, and superior frontal cortices. Within the cerebellum, a right-lateralized region 
also showed a significant effect of repetition. Several of these regions were also found to be more active for novel than for repeatedly presented items in separate comparisons of novel vs. repeated same and novel vs. repeated different. ${ }^{6}$

Direct comparison of the repeated different vs. repeated same conditions (see Table 2) showed greater activation (indicating less pronounced priming) for repeated different than repeated same items in several regions (see Fig. 3), including right precuneus and bilateral posterior inferior frontal, middle occipital, fusiform, parahippocampal (medial to $\mathrm{L}$ and $\mathrm{M}$ in Fig. 3 ) and superior parietal (not shown).

Examination of the time-courses of percent signal change for a subset of the regions that showed significant activation in the novel vs. all repeated comparison (particularly including frontal and occipitotemporal areas that were previously implicated in priming), showed that the level of activation typically reached peak amplitude four to six seconds following stimulus onset and returned to baseline by $14 \mathrm{~s}$. Representative timecourses are shown in Fig. 4 for peak activations in left and right posterior inferior frontal (BA 44, 6, shown as A, B in Fig. 3) and left and right fusiform cortex (BA 37, 19, shown as L, $\mathrm{M}$ in Fig. 3).

Analyses of variance performed on the peak amplitude response revealed that 4 out of 18 selected regions of interest (ROIs) did not show significant reductions for repeated different compared to novel items; these regions (i.e. brain foci that showed either a high level of perceptual specificity, or little 'abstract' visual priming, with no significant benefit achieved through prior exposure to a different exemplar of the object) were right fusiform ( $\mathrm{M}$ in Fig. 3), left and right precuneus ( $\mathrm{C}$ and $\mathrm{D}$ in Fig. 3), and right posterior inferior frontal cortex

\footnotetext{
${ }^{6}$ A complete listing of peak coordinates is available from the first author.
}

(cf. A and B in Fig. 3) 7 .

A region-of-interest analysis focusing on possible laterality differences in repetition priming for same vs. different exemplars in occipitotemporal cortex (right and left fusiform, Talairach coordinates of $46,-58$, -6 and $-40,-52,-6$, respectively) showed that there was a significantly greater effect of exemplar change in right than left fusiform cortex, $F(1,17)=$ 7.78, $M S E=0.001, P=0.01$ for the region $\times$ item type interaction. Although both regions showed less priming for repeated different than for repeated same exemplars, the modulation as a result of altered perceptual form was greater for right than left fusiform cortex (see Fig. 3, and time-course in Fig. 4).

\subsubsection{Repeated $>$ novel comparisons}

In addition to exploring the specificity of the neuroanatomical correlates of repetition priming, the current design also allows exploration of a somewhat different question: Do any brain regions show systematically greater activation for previously encountered objects than for novel items? Although the object classification task that participants performed did not

\footnotetext{
${ }^{7}$ This finding of no significant activation difference for novel objects compared with different object exemplars in right posterior inferior frontal cortex (BA 44) is broadly consistent with neuropsychological evidence and recent fMRI findings [see [30,63,65]] demonstrating material specificity effects in left vs. right prefrontal regions, such that right prefrontal areas are particularly recruited for the processing of nonverbal materials and left prefrontal areas for verbal materials. Indeed, to the extent that right prefrontal regions may also have been more sensitive to alterations in the precise perceptual form of objects than left prefrontal regions, an interaction of object type (same/different) and hemisphere (right/left) might have been expected. This was, in fact, a second planned interaction that we tested (Talairach coordinates of $-37,3,28$ and 40, 9, 31 for left and right, respectively); however, this interaction was not significant, $F<1$. (As with any null result, this finding should be interpreted cautiously.)
}

Fig. 3. Composite functional activation maps showing differences in brain activity for each of four comparisons: (1) novel $>$ all repeated (combining across repeated same and repeated different), (2) novel $>$ repeated same, (3) novel $>$ repeated different, (4) repeated different $>$ repeated same. Images are axial (horizontal) sections based on the averaged functional and anatomical data of 18 participants. The left side of each image corresponds to the left side of the brain. All regions shown passed the statistical threshold for significance. Coordinates on the inferior-superior $(z)$ axis from the stereotaxic atlas of Talairach and Tournoux [60] are shown at the bottom of the figure. In the comparison of novel $>$ all repeated, greater activation for novel than previously presented objects (reflecting priming) can be seen in several frontal and posterior and visual areas, including left and right posterior inferior frontal cortex (BA 44, 6, labeled A and B); left and right precuneus (BA 31, 19, labeled $\mathrm{C}$ and D); left and right frontal operculum (BA 45, 47, labeled E and F); left and right middle occipital (BA 19, labeled G and H); left and right posterior cingulate (BA 29, 30, labeled I and J); left anterior inferior frontal (BA 47, 45, labeled K) and left and right fusiform, extending into parahippocampal cortex (BA 37, 19, labeled L and M). Very similar regions are apparent in the separate comparison of novel $>$ repeated same. By contrast, a number of these regions were not significant in the novel $>$ repeated different comparison, including bilateral posterior inferior frontal (BA 44/6), bilateral precuneus (BA 31, 19), bilateral middle occipital (BA 19), and right fusiform (BA 37, 19). Particularly pronounced modulation by exemplar change can be seen in bilateral fusiform gyrus, especially the contrasting pattern of priming-related reductions in left (but not right) fusiform for novel $>$ different (Panel 3) as opposed to greater activation in right fusiform in the comparison of different $>$ same (Panel 4). 
Table 2

Regions demonstrating greater activation for repeated different than repeated same objects ${ }^{\mathrm{a}}$

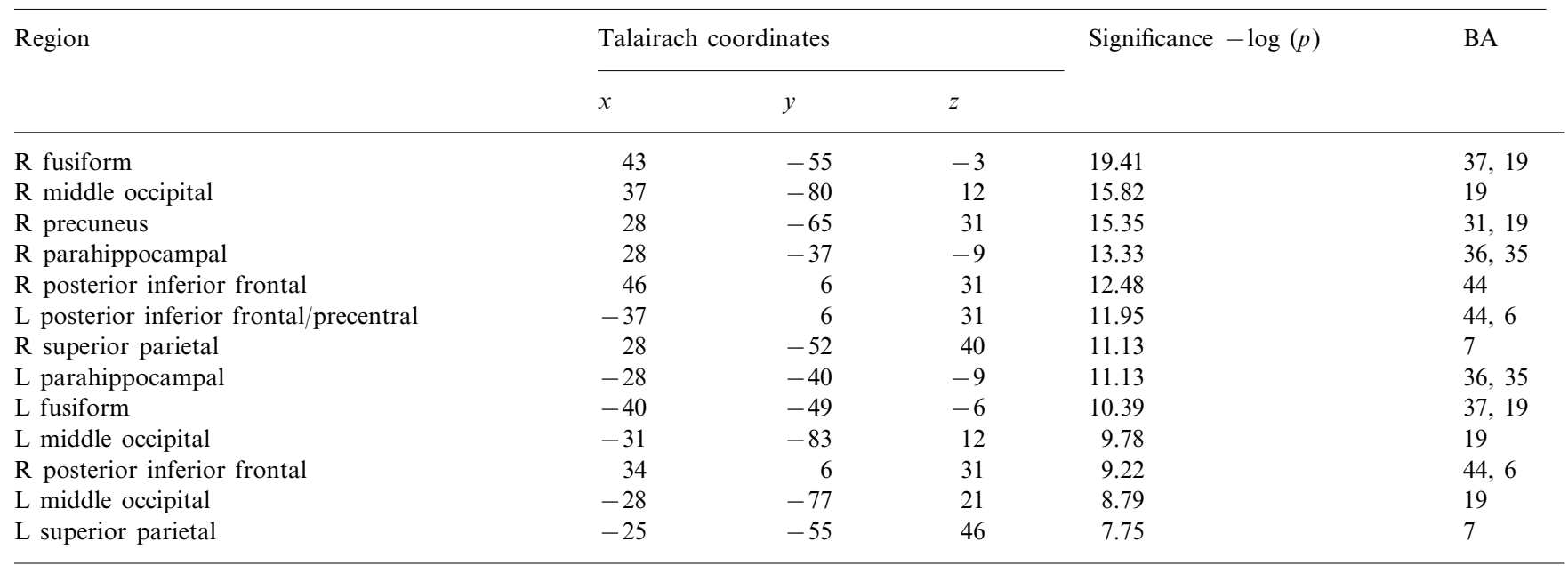

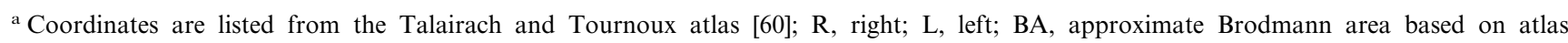
coordinates.

require explicit recollection of the previously presented items (participants were simply asked to repeatedly judge the size of the referents of the pictured objects), processing of the repeated items might nonetheless yield greater activation than for novel items either because: (a) repeated items might prompt recall of the item's earlier presentation, with such recall possibly occurring intentionally, but also possibly reflecting 'incidental' or 'involuntary' remembering in the absence of an explicit intention to retrieve or of any deliberate search [cf. $[1,46,47,53,56]]$ or (b) some brain regions might show greater activation for 'old' than 'new' items, even though this 'old/new' effect is not correlated with remembering per se. In either case, activation in brain regions that have been found to be involved in memory retrieval (e.g., recognition or recall) or that show 'old > new' effects might be expected.

To explore this secondary question, we also performed analyses that examined whether activation was greater for all repeated objects than for novel objects. Several parietal, cingulate, and temporal activations were found in this comparison (see Table 3), and also in a separate comparison of repeated same $>$ novel. However, in the latter comparison, several further foci were observed that were not apparent for all repeated $>$ novel, including right and left frontal activations, particularly in middle frontal regions (BA 10, BA 9, BA 8, 8/6); bilateral superior temporal activations (BA 42, 42/22) were also seen. Direct comparison of the repeated same and repeated different items showed many similar activations; Table 4 lists activations in frontal regions that emerged in this comparison. ${ }^{8}$

\footnotetext{
${ }^{8}$ Complete listings of these activations, and those for the repeated same $>$ novel comparison, are available from the first author upon request.
}

ROIs were examined, including bilateral parietal regions showing an all repeated $>$ novel pattern, bilateral parietal, frontal, and left superior temporal regions showing a repeated same $>$ novel pattern, and several frontal regions showing a repeated same $>$ repeated different pattern. In the parietal regions, the timecourses demonstrated signal reductions such that the activation level for the object trials fell below the fixation baseline, with the repeated same items showing more modest reductions relative to the repeated different or novel items. By contrast, several of the frontal regions showed an actual 'activation increase' for the repeated same items. For the repeated same $>$ novel comparison, these included bilateral middle frontal cortex (BA 10: 25, 53, 15 and $-25,56,18$; also BA 46/9: $-21,44,31)$, and a more posterior region, near middle frontal gyrus (BA 6 and BA 8: 37, 6, 53 and 40, 16, 46). Left superior temporal cortex $(-56,-15,6)$ likewise showed an activation increase. For the repeated same $>$ repeated different comparison, a left middle frontal region (BA 10: $-25,59,15)$, and a region in cuneus/superior occipital cortex (BA 19: $-3,-80$, 34), showed activation increases.

Random effects analyses on the eight ROIs that showed an actual activation increase for the repeated same items relative to the novel or repeated different items revealed, in each instance, a significant effect of item type (smallest $F(2,34)=4.48$ ), and significantly greater activation for repeated same than novel, and for repeated same than repeated different. By contrast, for six of these regions, repeated different and novel items showed no reliable difference in amplitude of activation (and one region showed significantly less activation for repeated different than for novel).

Although it is not possible to determine whether these greater activations for repeated items than for 


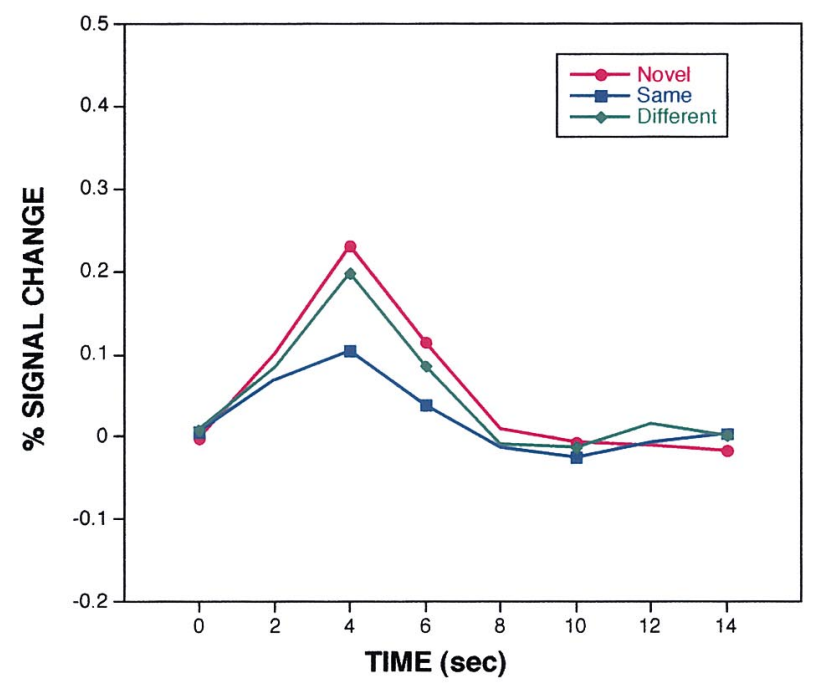

L Fusiform: $-40,-52,-6$ (BA 37, 19)

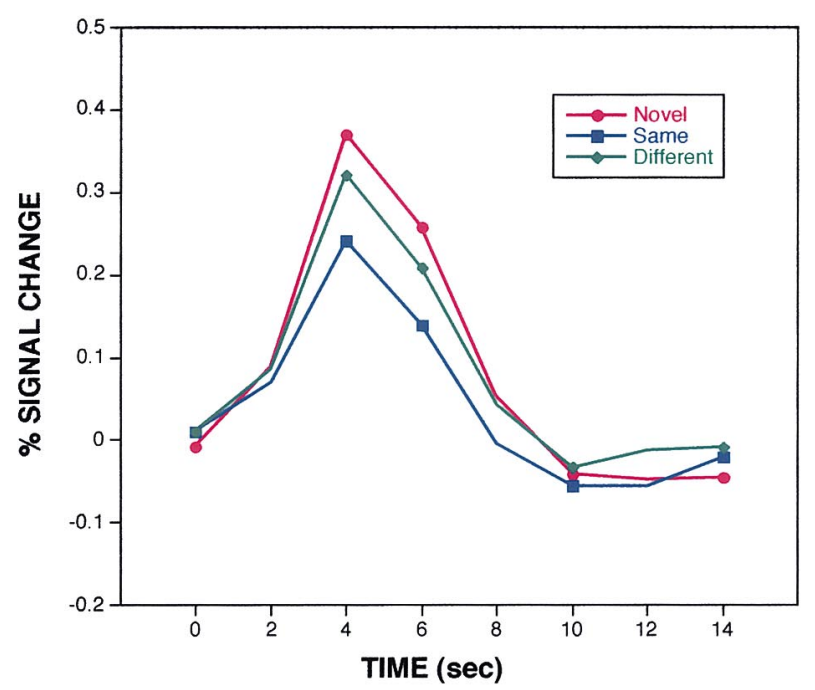

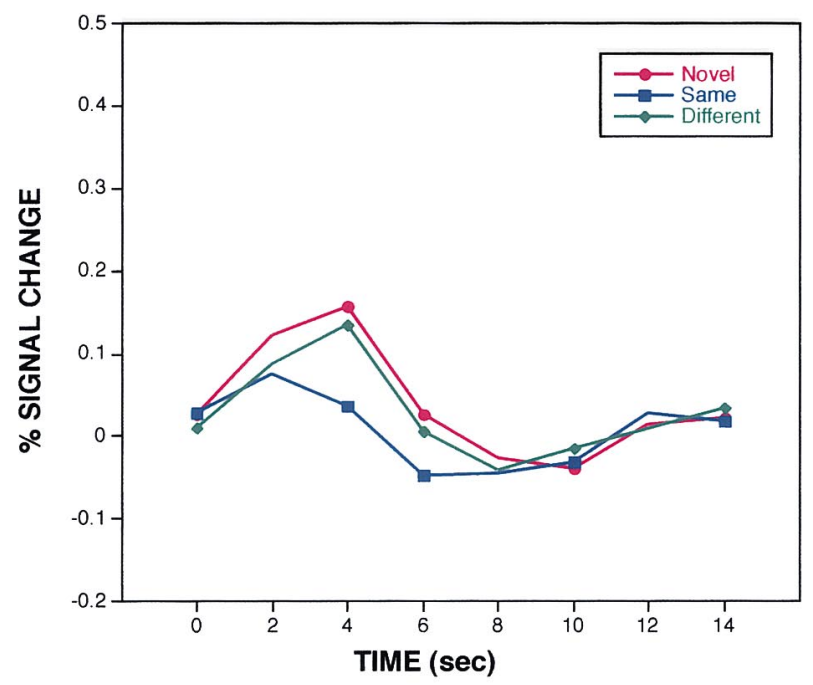

R Fusiform: 46, -58, -6 (BA 37, 19)



Fig. 4. Percent fMRI signal change (relative to the visual fixation baseline) for novel, repeated same, and repeated different objects in left and right posterior inferior frontal cortex (BA 44, 6, peak ROI coordinates of $-37,3,28$ and 40, 9, 31, respectively), and for left and right fusiform cortex (BA 37, 19; peak ROI coordinates of $-40,-52,-6$ and 46, $-58,-6$, respectively), shown in the upper and lower panel respectively. The interaction between laterality and perceptual similarity across repetitions was significant for the regions of fusiform gyrus.

novel items reflect intentional or incidental recollection, or old $>$ new effects unrelated to memory per se, here it might briefly be noted - especially as an observation for future studies - that three regions, in particular, appear to nearly match activations that have been previously reported in recognition memory paradigms. One of these regions demonstrated a reduction (followed by an increase) relative to the fixation baseline: 1. Inferior parietal (BA 40, all repeated $>$ novel: -40 , $-58,40)$. Here observed bilaterally, activation in this region was reported particularly on the left in a comparison of hits vs. correct rejections by Konishi et al. [31] ( $-39,-55,36$; also $33,-53,44)$, and in a comparison of correct 'remember' responses relative to correct 'new' responses by Henson et al. [28] (superior parietal: $-33,-60,45$ ). Also, in a quantitative meta-analysis of five PET studies examining recognition memory, Habib and Lepage [25] reported a similar region $(-36,-58,32)$ as showing 'familiarity activations', that is, greater activation 
Table 3

Regions demonstrating greater activation for repeated than novel objects (combining across same and different objects) ${ }^{\mathrm{a}}$

\begin{tabular}{|c|c|c|c|c|c|}
\hline \multirow[t]{2}{*}{ Region } & \multicolumn{3}{|c|}{ Talairach coordinates } & \multirow[t]{2}{*}{ Significance $-\log (p)$} & \multirow[t]{2}{*}{ BA } \\
\hline & $x$ & $y$ & $z$ & & \\
\hline Precuneus & 6 & -62 & 40 & 26.92 & 7 \\
\hline Posterior cingulate & 0 & -21 & 34 & 15.29 & 23 \\
\hline $\mathrm{L}$ inferior parietal & -40 & -58 & 40 & 13.36 & 40 \\
\hline L superior parietal & -34 & -65 & 50 & 8.52 & 7 \\
\hline
\end{tabular}

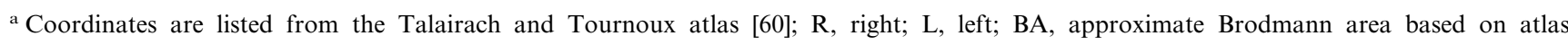
coordinates.

for familiar stimuli, including pictures, words, and auditory sentences, than for new items.

In addition, two regions manifested actual activation increases for the repeated same items:

2. Middle frontal gyrus (BA 10, repeated same $>$ repeated different: $-25,59,15)$. Here observed bilaterally, activation in this region was found particularly on the left in the comparisons of the prior studies noted above (Konishi et al.: - 35, 51, 8; Habib and Lepage: - 40, 44, 20; Henson et al., superior frontal: $-12,63,18)$.

3. Medial cuneus/superior occipital (BA 19: $-3,-80$, 34). Here found in the repeated same $>$ repeated different comparison, this region is very close to one reported in the meta-analysis of Habib and Lepage (BA 19: 6, - 76, 32) as demonstrating greater activation for familiar stimuli than for new items; it is also close to a region reported by Konishi et al. ( -7 , $-73,34)$ for hits vs. correct rejections, and Henson et al. $(0,-66,33)$ for correct 'remember' vs. 'new' responses.

\section{Discussion}

Consistent with several previous studies [e.g. [9,64]], we found priming-related reductions in neural activity when participants performed classification judgments for previously presented objects relative to novel objects. Reduced neural activity for repeated objects was observed in multiple frontal regions, including left anterior inferior frontal (BA 47, 45) and left posterior inferior frontal (BA $44,6)$ cortex - possibly reflecting increased ease in accessing and/or working with semantic or phonological attributes of recently processed items [e.g. [10,16,20,44]] - as well as right posterior inferior frontal cortex (BA 44) - possibly reflecting decreased demands on visuospatial attentional processes required for the size judgment task [e.g. [30,63]] for repeated items.

Priming-related reductions in neural activity were also seen in multiple posterior and higher-order visual areas, including bilateral middle occipital (BA 19), parahippocampal (BA 36/35) and fusiform (BA 37/19) cortices. These reductions, largely paralleling those found in a previous fMRI study [9] using a similar experimental paradigm but including only repeated same object exemplars may - in part (see further discussion below) - reflect perceptual processing benefits achieved from repeated processing of the same stimulus. These priming-related reductions for novel vs. repeated objects, observed here using fMRI, may also be similar to reductions in intracranial event-related potentials, recently reported by Puce, Allison, and McCarthy [43] for repeatedly presented faces. Recording from electrodes placed directly on the cortical surface of the brain (to monitor intractable seizures), these researchers found substantial and progressive decreases in the early portion of an N700 potential and in a P350 potential at face-specific sites, showing increasing habituation across repeated presentations of initially novel faces (the faces were presented eight times in a blocked, nonintermixed fashion and results were similar for right and left hemisphere sites). At the neuronal level, these priming-related reductions may also parallel the phenomenon, observed using single-unit recordings in non-human primates and variously designated as 'repetition suppression', 'stimulusspecific adaptation', and 'adaptive mnemonic filtering', demonstrating reduced neuron activity for repeatedly exposed objects and faces compared with the initial or novel presentation of those stimuli [e.g. [33,41,48,51]].

Critically, in addition to these overall (novel vs. repeated) priming effects, we found that the magnitude of facilitation observed in bilateral middle occipital (BA 19), parahippocampal (BA 36/35) and fusiform (BA $37 / 19$ ) cortices was modulated by alterations in the perceptual form of the object. Comparatively more modest reductions in neural activity were found for different exemplars of the earlier exposed objects than for the same exemplars. Furthermore, a region of occipitotemporal (fusiform) cortex showed laterality differences in sensitivity to alterations in the perceptual form 
Table 4

Frontal regions demonstrating greater activation for repeated same than for repeated different exemplars ${ }^{\mathrm{a}}$

\begin{tabular}{|c|c|c|c|c|c|}
\hline \multirow[t]{2}{*}{ Region } & \multicolumn{3}{|c|}{ Talairach coordinates } & \multirow[t]{2}{*}{ Significance $-\log (p)$} & \multirow[t]{2}{*}{ BA } \\
\hline & $x$ & $y$ & $z$ & & \\
\hline $\mathrm{R}$ middle frontal & 28 & 56 & 15 & 13.83 & 10 \\
\hline Medial frontal & 6 & 53 & 6 & 11.31 & 10 \\
\hline $\mathrm{R}$ middle frontal & 21 & 19 & 46 & 10.17 & 8 \\
\hline $\mathrm{L}$ middle frontal & -21 & 22 & 40 & 9.02 & 8 \\
\hline $\mathrm{R}$ middle frontal & 28 & 34 & 37 & 8.59 & 8,9 \\
\hline Superior frontal & 3 & -15 & 59 & 8.52 & 6 \\
\hline $\mathrm{R}$ middle frontal & 37 & 9 & 43 & 8.51 & 6,8 \\
\hline $\mathrm{R}$ superior frontal & 9 & -12 & 71 & 7.91 & 6 \\
\hline
\end{tabular}

${ }^{a}$ Coordinates are listed from the Talairach and Tournoux atlas [60]; R, right; L, left; BA, approximate Brodmann area based on atlas coordinates.

of repeated objects. Specifically, visual form changes were associated with a larger 'cost' to the neural benefits achieved through prior experience in right (Talairach coordinates of $46,-58,-6)$ relative to left $(-40,-52$, -6) fusiform cortex. This latter finding is broadly consistent with behavioral findings from divided visual field studies that have shown greater decrements in repetition priming for perceptually altered stimuli presented directly to the right than to the left cerebral hemisphere ${ }^{9}$, and with neuropsychological evidence from prosopagnosic patients, suggesting a possible differential involvement of right occipitotemporal cortices in allowing discrimination of categorically related objects. The absence of font-specific priming reported by Vaidya et al. [62] in patient M.S. following surgical resection of right occipital cortex is likewise broadly consistent with this finding. (Also see Davidoff and Warrington [15], for a patient with a predominantly right hemisphere temporoparieto-occipital lesion who showed extremely impaired

\footnotetext{
${ }^{9}$ One, apparently inconsistent, observation between the present findings and those obtained using the divided visual field paradigm concerns the exact stimulus presentation parameters under which specificity may be observed. Whereas in the present study we observed hemispheric differences in specific (or abstract) priming using a 500-ms exposure of the objects at study and at test, Marsolek [35], using line-drawings of common objects in a divided visual field procedure, failed to find such a specificity difference for a $500-\mathrm{ms}$ exposure but did find a specificity difference using a longer exposure time (three seconds). There are numerous methodological differences between the behavioral divided visual field paradigm and the current study that might contribute to this differing pattern [e.g. the stimuli in the present experiment were detailed colored objects and repeated items were presented multiple times before the critical test trial, but participants performed the same orienting task (size judgments) for the objects on each presentation; in contrast, the stimuli in the divided visual field study were black and white line drawings, participants performed a different task during the initial exposure than during the test trial (one of three different orienting tasks vs. confrontation naming on the test trial), and objects at test were presented either to the right or left visual field for only $17 \mathrm{~ms}$ each.]
}

object recognition for objects presented in nonconventional but not conventional views, and who was largely unable to detect alterations in color, shape, or parts of objects that he could, nevertheless, correctly name.)

The region of left fusiform gyrus (Talairach coordinates of $-40,-52,-6$ ) that showed less sensitivity to the perceptual change between different and same exemplars (as indexed by less of a reduction in priming) is close to a region that has shown greater activation during the encoding of visually presented words that were subsequently remembered relative to those that were subsequently forgotten (coordinates of $-43,-55,-9$ ) [66], suggesting that activation in this region is not specific to visual object forms. Moreover, we have also recently observed repetition-related reductions in left fusiform activity using a word generation task in which participants were asked to covertly generate words (e.g. participant encounters ' $\mathrm{COU}{ }_{--}$' and generates 'courage') in response to visually (coordinates of $-50,-58,-12$ ) or auditorily presented (coordinates of $-43,-52$, -12) 'word stems' [[10]; see also [1]].

In view of these latter outcomes, it might be necessary to revise earlier interpretations of priming-related reductions in this region that attributed facilitation to enhanced visual perceptual processing [54,70]. Rather, because similar reductions in this region were observed for visual word stems and auditory word-stems where no visual form was presented, the reductions might reflect facilitation of non-visual processing involved in the wordgeneration task. Evidence congruent with this interpretation includes other reports of activation in this region during auditory presentation of words [67] and findings from presurgical explorations in epileptic patients [34] suggesting that this region - possibly close to, or overlapping with, a region that has been termed the 'basal temporal language area' - may assume an important role in linking meaning with words [67], or in 
naming or tagging stimuli that are recognizable [[6]; also see [45]]. Consistent with this possibility, recent PET evidence [42] indicates that patients with semantic dementia, who show especially pronounced difficulties in naming, demonstrate less activation in this region (coordinates of $-54,-52,-10)$ than do normal controls during semantic comparison tasks involving both pictures and words.

On this interpretation, although the object classification task used in the present experiment did not require generating the names of the pictured objects, some lexical processing may have occurred. Activation of left inferior frontal gyrus, consistently found during tasks involving lexical/semantic elaboration, is congruent with this possibility. If the 'mapping' of lexical, conceptual, or phonological information to the objects was relatively similar for the two exemplars of an object pair, then greater transfer (facilitation) would be expected across object exemplars for this information (shown in relatively preserved 'neural priming' in left fusiform cortex for different exemplars) than for visual form information, which was deliberately varied across exemplars (shown in more marked reductions in neural priming in right fusiform). Evidence of some (albeit less marked) modulation in priming for different - compared to same exemplar objects in left fusiform might have arisen if the exemplars emphasized somewhat different conceptual or semantic features of the objects, and thus affected the mapping between pictorial form and lexical/phonological information, or led to differences in how the objects within an exemplar pair were named. [Note that the normative behavioral data are also consistent with this account, with participants who were asked to directly name the objects providing 'non-identical' names for the two exemplars within a pair for a total of about $18 \%$ of the items - $10 \%$ comprising minor variants such as flower vs. flowers; bunny vs. bunny rabbit; gift vs. gift box; and $8 \%$ comprising different levels of classification, such as bird vs. parrot; cookies vs. oreos, but with variations of both types tending to emerge for different items for different individuals.]

Alternative accounts of the differential modulation of the right and left occipitotemporal regions, as a function of perceptual specificity, are also possible. The left fusiform might also be partially sensitive to perceptual form, but relatively less so than right fusiform, or it may operate at a more 'abstract' level of visual form. This latter proposal may be closer to that forwarded by Marsolek [35] to account for differential effects of perceptual specificity in divided visual-field studies. Marsolek [35] has specifically argued that the dissociable subsystem underlying abstract-category object recognition in left posterior regions is a visual subsystem — 'one that processes only visual-structure information' (p. 111) - rather than arising from the similar treatment of different exemplars in post-visual phonological or con- ceptual subsystems (though such post-visual subsystems may provide the feedback that then allows the visual subsystem to map abstract visual inputs to the same name or output representation). Marsolek ([35]; see also [38]) has suggested that greater sensitivity to alterations of perceptual form in the right hemisphere may reflect a relatively more holistically-based visual processing strategy (with features not represented independently in the subsystem) whereas left hemisphere processing may be relatively more feature-based (with the different features of inputs represented separately in the subsystem, thereby allowing classification on the basis of subsets of similar features).

On the one hand, the outcomes of the present experiment appear entirely consistent with Marsolek's proposal. On the other hand, at least for the particular regions of fusiform cortex that here demonstrated differential modulation as a function of exemplar type, an account strictly focusing on visual object forms does not appear to adequately explain the priming-related reductions that we found in a very similar region of left fusiform during processing of words and also auditory word-stems, where no visual form information was presented, nor would it provide an account of the apparent similarity of these findings to outcomes regarding the 'basal temporal language area' (reviewed above). Nonetheless, it remains possible that differences in specific-exemplar vs. abstract-category processing for strictly visual-structure information are also present, but in a region other than the fusiform region that here showed an object-type $\times$ hemisphere interaction, or that the regions found in the word-stem completion and object priming studies are not identical. It is also possible that the fusiform region that we here observed to be differentially affected by repeated-same compared with repeated-different object exemplars subserves, or is part of, an abstract visual word form system rather than an abstract visual object form system. (Marsolek's distinction of abstract-category vs. specific-exemplar based processing also allows for this possibility, proposing that the left cerebral hemisphere implements, at least in part, a subsystem that recognizes abstract categories of various kinds of visual forms, including word forms as well as other shapes.) An abstract visual word form system could then reasonably contribute to all of the tasks in which modulation of this region has been observed: (i) encoding of visually presented words (with greater activation of the abstract visual word form associated with later remembering rather than forgetting, [66]), (ii) encoding and generation of completions in the auditory word-stem completion task (recoding the word into an abstract word form or into a subword component to allow the covert generation of a completion, or possibly as a consequence of such generation, [10]), and (iii) in the current task recoding of the pictures into names or into a conceptual/ phonological form, either with conscious awareness or 
without such awareness, but with such recoding not always identical across the exemplars of a pair.

To begin to resolve these issues, future experiments could examine the effects of perceptual similarity manipulations under conditions where the objects are non-representational abstract forms, without any pre-existing lexical or semantic associations, or where the visual forms are manipulated to repeat relatively more 'abstract' levels of visual form (e.g. a lollipop and a magnifying glass may share similar overall shape, cf. [27]) but where the lexical and semantic information associated with those similar abstract forms clearly differs. On an abstract visual object-form account, such similarly-shaped but differently-named items might result in priming-related reductions in neural activity across objects of similar shape (particularly in left fusiform cortex). By contrast, on the basis of an account emphasizing abstract lexical, phonological, or conceptual codes involved in naming or otherwise tagging stimuli, this condition should not yield evidence of priming because the names associated with the similar shapes are different. (An abstract visual word-form account would yield predictions similar to this second alternative.) Yet a further possibility might be to examine the consequences of providing or not providing the object's name prior to the presentation of the objects: if the residual differences that we observed in left fusiform gyrus for same vs. different exemplars arose because at least in some instances - different 'lexical tags' were evoked by the two exemplars, then ensuring that the same 'tag' is evoked (by providing it) should reduce, and possibly eliminate, the effect of exemplar type (same vs. different) in left fusiform; however, if the difference was primarily driven by visual object-form differences, then provision of the common (shared) name should have little effect and a difference in the magnitude of priming for same vs. different exemplars (same $>$ different) in left fusiform gyrus should still be found.

Finally, some possible questions regarding the generalizability of the present findings - beyond the specific task conditions that were used in this study - should be noted. First, the encoding task that we used, involving comparative size judgments of objects, comprises a relatively higher order visual-semantic task and an attribute that is inferred, rather than directly present, in the objects [cf. [26]]. The extent to which a similar pattern of perceptual specificity might be obtained for other, possibly lower level or directly presented perceptual or sensory attributes, or for abstract attributes with no explicit perceptual component (e.g. monetary value), is not known.

Second, the precise nature of the perceptual changes for the same and different exemplars that might be sufficient to observe modulation of the 'neural priming' effect cannot, of course, be determined from this study where - in order to maximize our initial likelihood of finding both neural and behavioral differences for same and different exemplars - multiple aspects of the stimuli (as for the different exemplars of everyday objects) were varied, including shape, color, surface pattern, material, and (to a lesser extent) orientation. Behavioral studies of perceptual specificity effects in repetition priming suggest that some of these factors are likely to be more influential in determining behavioral differences than others. For example, using a picture naming task, Cave et al. [12] found no effects of manipulations of color or texture on naming latency (even though these changes could be detected at abovechance levels, they did not detrimentally affect naming response times). Nonetheless, several aspects made the different exemplars of the present study perceptually different, and thus may have contributed to the behavioral and neural patterns observed.

Third, there are questions concerning the possible mediating role of attention to the perceptual form changes and the relative saliency and task-relevance of those changes. Insofar as, in the present study, item exemplar pairs were selected such that they yielded the same 'answer' for both of the exemplars within a pair, and same/different exemplar status was not relevant to the size judgment task that participants were asked to perform, it is possible that the emergence of neural perceptual specificity effects does not depend on participants' explicit attentional focus on the varying perceptual nature of the exemplars. However, it is also quite possible that the specific task conditions that we employed, such as the number of initial repetitions of the items, or the repeated study-test cycle format, acted to especially enhance 'item-specific' perceptual information for the presented items and/or to increase participants' awareness of the altered perceptual form of the different exemplars - perhaps encouraging participants to adopt something akin to a 'stimulus discrimination' approach. The extent to which these factors may modulate the magnitude or the pattern of neural priming effects in relation to perceptual specificity is not known [compare, for example, [13,49,57]]. Efforts to examine these questions are likely to continue to yield insights into the ways that we benefit from prior encounters with objects, the brain regions that support such learning, and the nature of perceptual specificity effects in object processing. They might also illuminate the more difficult to define complementary pole of visual specificity, denoted by the term 'abstract visual form', whereby — despite considerable variations in perceptual appearances - we may still judge that 'a cup, is a cup, is a cup'.

\section{Acknowledgements}

This research was supported by NIA AG08441, NIMH MH57506, NIH MH60941 the Human Fron- 
tiers Science Program, and the McDonnell Center for Higher Brain Function. We thank Chandan Reddy for assistance in collecting and formatting the stimuli, and Sara Greene for help in behavioral piloting and collection of normative data.

\section{References}

[1] Badgaiyan RD, Schacter DL, Alpert NM. Auditory priming within and across modalities: evidence from positron emission tomography. Journal of Cognitive Neuroscience 1999;11:337-48.

[2] Bartram DJ. The role of visual and semantic codes in object naming. Cognitive Psychology 1974;6:325-56.

[3] Biederman I, Cooper EE. Size invariance in visual object priming. Journal of Experimental Psychology: Human Perception and Performance 1992;18:121-33.

[4] Biederman I, Gerhardstein PC. Recognizing depth-rotated objects: evidence and conditions for three-dimensional viewpoint invariance. Journal of Experimental Psychology: Human Perception and Performance 1993;19:1162-82.

[5] Blaxton TA, Bookheimer SY, Zeffiro TA, Figlozzi CM, Gaillard WD, Theodore WH. Functional mapping of human memory using PET: comparisons of conceptual and perceptual tasks. Canadian Journal of Experimental Psychology 1996;50:42-56.

[6] Bookheimer SY, Zeffiro TA, Blaxton T, Gaillard W, Theodore W. Regional cerebral blood flow during object naming and word reading. Human Brain Mapping 1995;3:93-106.

[7] Bornstein B, Sroka H, Munitz H. Prosopagnosia with animal face agnosia. Cortex 1969;5:164-9.

[8] Buckner RL, Petersen SE, Ojemann JG, Miezin FM, Squire LR, Raichle ME. Functional anatomical studies of explicit and implicit memory retrieval tasks. Journal of Neuroscience 1995; 15:12-29.

[9] Buckner RL, Goodman J, Burock M, Rotte M, Koutstaal W, Schacter DL, et al. Functional-anatomic correlates of object priming in humans revealed by rapid presentation event-related fMRI. Neuron 1998;20:285-96.

[10] Buckner RL, Koutstaal W, Schacter DL, Rosen BR. Functional MRI evidence for a role of frontal and inferior temporal cortex in amodal components of priming. Brain 2000;123:620-40.

[11] Burock MA, Buckner RL, Woldorff MG, Rosen BR, Dale AM. Randomized event-related experimental designs allow for extremely rapid presentation rates using functional MRI. NeuroReport 1998;9:3735-9.

[12] Cave CB, Bost PR, Cobb RE. Effects of color and pattern on implicit and explicit picture memory. Journal of Experimental Psychology: Learning, Memory, and Cognition 1996;22:639-53.

[13] Corbetta M, Miezin FM, Dobmeyer S, Shulman GL, Petersen SE. Selective and divided attention during visual discriminations of shape, color, and speed: functional anatomy by positron emission tomography. Journal of Neuroscience 1991;11:2383402.

[14] Dale AM, Buckner RL. Selective averaging of rapidly presented individual trials using fMRI. Human Brain Mapping 1997;5:329-40.

[15] Davidoff J, Warrington EK. The bare bones of object recognition: implications from a case of object recognition impairment. Neuropsychologia 1999;37:279-92.

[16] Demb JB, Desmond JE, Wagner AD, Vaidya CJ, Glover GH, Gabrieli JDE. Semantic encoding and retrieval in the left inferior prefrontal cortex: a functional fMRI study of task difficulty and process specificity. Journal of Neuroscience 1995;15:5870-8.

[17] De Renzi E. Prosopagnosia: a multi-stage, specific disorder. In: Young AW, Ellis HD, editors. Handbook of research on face processing. Amsterdam: North-Holland, 1989:27-35.
[18] Farah MJ. Patterns of co-occurrence among the associative agnosias: implications for visual object representation. Cognitive Neuropsychology 1991;8:1-19.

[19] Gabrieli JDE. Cognitive neuroscience of human memory. Annual Review of Psychology 1998;49:87-115.

[20] Gabrieli JDE, Desmond JE, Demb JE, Wagner AD, Stone MV, Vaidya CJ, et al. Functional magnetic resonance imaging of semantic memory processes in the frontal lobes. Psychological Science 1996;7:278-83.

[21] Gabrieli JDE, Fleischman DA, Keane MM, Reminger SL, Morrell F. Double dissociation between memory systems underlying explicit and implicit memory in the human brain. Psychological Science 1995;6:76-82.

[22] Gauthier I, Anderson AW, Tarr MJ, Skudlarski P, Gore JC. Levels of categorization in visual recognition studies using functional magnetic resonance imaging. Current Biology 1997;7:64551.

[23] Gauthier I, Behrmann M, Tarr MJ. Can face recognition really be dissociated from object recognition? Journal of Cognitive Neuroscience 1999;11:349-70.

[24] Graf P, Ryan L. Transfer-appropriate processing for implicit and explicit memory. Journal of Experimental Psychology: Learning, Memory, and Cognition 1990;16:978-92.

[25] Habib R, Lepage M. Novelty assessment in the brain. In: Tulving E, editor. Memory, consciousness, and the brain: the Tallinn Conference. Philadelphia, PA: Taylor \& Francis, 2000:265-77.

[26] Hart JA, Jr., Lesser RP, Gordon B. Selective interference with the representation of size in the human by direct cortical electrical stimulation. Journal of Cognitive Neuroscience 1992;4:33744.

[27] Henkel LA, Franklin N. Reality monitoring of physically similar and conceptually related objects. Memory \& Cognition 1998;26:659-73.

[28] Henson RNA, Rugg MD, Shallice T, Josephs O, Dolan RJ. Recollection and familiarity in recognition memory: an event-related functional magnetic resonance imaging study. Journal of Neuroscience 1999;19:3962-72.

[29] Jolicoeur P, Gluck M, Kosslyn SM. Pictures and names: making the connection. Cognitive Psychology 1984;16:243-75.

[30] Kelley WM, Miezin FM, McDermott KB, Buckner RL, Raichle ME, Cohen NJ, et al. Hemispheric specialization in human dorsal frontal cortex and medial temporal lobe for verbal and nonverbal memory encoding. Neuron 1998;20:927-36.

[31] Konishi S., Wheeler M.E., Donaldson D.I., Buckner R.L. Neural correlates of episodic retrieval success. NeuroImage. 2000;12: 276-86.

[32] Kosslyn SM, Alpert NM, Thompson WL. Identifying objects at different levels of hierarchy: A positron emission tomography study. Human Brain Mapping 1995;3:107-32.

[33] Li L, Miller EK, Desimone R. The representation of stimulus familiarity in anterior inferior temporal cortex. Journal of Neurophysiology 1993;69:1918-29.

[34] Luders H, Lesser RP, Hahn J, Dinner DS, Morris HH, Wyllie E, et al. Basal temporal language area. Brain 1991;114:743-54.

[35] Marsolek CJ. Abstract visual-form representations in the left cerebral hemisphere. Journal of Experimental Psychology: Human Perception and Performance 1995;21:375-86.

[36] Marsolek CJ. Dissociable neural subsystems underlie abstract and specific object recognition. Psychological Science 1999;10:111-8.

[37] Marsolek CJ, Kosslyn SM, Squire LR. Form-specific visual priming in the right cerebral hemisphere. Journal of Experimental Psychology: Learning, Memory, and Cognition 1992;18:492508.

[38] Marsolek CJ, Schacter DL, Nicholas CD. Form-specific visual priming for new associations in the right cerebral hemisphere. Memory \& Cognition 1996;24:539-56. 
[39] Marsolek CJ, Squire LR, Kosslyn SM, Lulenski ME. Form-specific explicit and implicit memory in the right cerebral hemisphere. Neuropsychology 1994;8:588-97.

[40] Martin A, LaLonde FM, Wiggs CL, Weisberg J, Ungerleider LG, Haxby JV. Repeated presentation of objects reduces activity in ventral occipitotemporal cortex: A fMRI study of repetition priming. Society for Neuroscience Abstracts 1995;21:1497.

[41] Miller EK, Desimone R. Parallel neuronal mechanisms for short-term memory. Science 1994;263:520-2.

[42] Mummery CJ, Patterson K, Wise RJS, Vandenbergh R, Price CJ, Hodges JR. Disrupted temporal lobe connections in semantic dementia. Brain 1999;122:61-73.

[43] Puce A, Allison T, McCarthy G. Electrophysiological studies of human face perception. III: effects of top-down processing on face-specific potentials. Cerebral Cortex 1999;9:445-58.

[44] Raichle ME, Fiez JA, Videen TO, MacLeod AM, Pardo JV, Fox PT, et al. Practice-related changes in human brain functional anatomy during nonmotor learning. Cerebral Cortex 1994;4:826.

[45] Raymer AM, Foundas AL, Maher LM, Greenwald ML, Morris M, Rothi LJG, et al. Cognitive neuropsychological analysis and neuroanatomic correlates in a case of acute anomia. Brain and Language 1997;58:137-56.

[46] Richardson-Klavehn A, Gardiner JM, Java RI. Involuntary conscious memory and the method of opposition. Memory 1994;2:1-29.

[47] Richardson-Klavehn A, Lee MG, Joubran R, Bjork R. Intention and awareness in perceptual identification priming. Memory \& Cognition 1994;22:293-312.

[48] Riches IP, Wilson FAW, Brown MW. The effects of visual stimulation and memory on neurons of the hippocampal formation and the neighboring parahippocampal gyrus and inferior temporal cortex of the primate. Journal of Neuroscience 1991;11:1763-79.

[49] Richmond BJ, Sato T. Enhancement of inferior temporal neurons during visual discrimination. Journal of Neurophysiology 1987;58:1292-306.

[50] Roediger HL III, McDermott KB. Implicit memory in normal human subjects. In: Spinnler H, Boller F, editors. Handbook of neuropsychology, vol. 8. Amsterdam: Elsevier, 1993:63-131.

[51] Rolls ET, Baylis GC, Hasselmo ME, Nalwa V. The effect of learning on the face selective responses of neurons in the cortex in the superior temporal sulcus of the monkey. Experimental Brain Research 1989;76:153-64.

[52] Rosch E, Mervis CB, Gray WD, Johnson DM, Boyes-Braem P. Basic objects in natural categories. Cognitive Psychology $1976 ; 8: 382-439$

[53] Schacter DL, Badgaiyan RD, Alpert NM. Visual word stem completion priming within and across modalities: a PET study. NeuroReport 1999;10:2061-5.
[54] Schacter DL, Buckner RL. Priming and the brain. Neuron 1998;20:185-95.

[55] Schacter DL, Alpert NM, Savage CR, Rauch SL, Albert MS. Conscious recollection and the human hippocampal formation: evidence from positron emission tomography. Proceedings of the National Academy of Sciences, USA 1996;93:321-5.

[56] Schacter DL, Bowers J, Booker J. Intention, awareness, and implicit memory: The retrieval-intentionality criterion. In: Lewandowsky S, Dunn JC, Kirsner K, editors. Implicit memory: theoretical issues. Hillsdale, NJ: Erlbaum, 1989:47-65.

[57] Spitzer H, Richmond BJ. Task difficulty: ignoring, attending to, and discriminating a visual stimulus yield progressively more activity in inferior temporal neurons. Experimental Brain Research 1991;83:340-8.

[58] Squire LR, Ojemann JG, Miezin FM, Petersen SE, Videen TO, Raichle ME. Activation of the hippocampus in normal humans: A functional anatomical study of memory. Proceedings of the National Academy of Sciences, USA 1992;89:1837-41.

[59] Srinivas K. Size and reflection effects in priming: a test of transfer-appropriate processing. Memory \& Cognition 1996;24:441-52.

[60] Talairach J, Tournoux P. Co-planar stereotaxic atlas of the human brain. New York: Thieme, 1988.

[61] Tanaka JW, Taylor M. Object categories and expertise: Is the basic level in the eye of the beholder? Cognitive Psychology 1991;23:457-82.

[62] Vaidya CJ, Gabrieli JDE, Verfaellie M, Fleischman D, Askari N. Font-specific priming following global amnesia and occipital lobe damage. Neuropsychology 1998;12:183-92.

[63] Wagner AD. Working memory contributions to human learning and remembering. Neuron 1999;22:19-22.

[64] Wagner AD, Desmond JE, Demb JB, Glover GH, Gabrieli JDE. Semantic repetition priming for verbal and pictorial knowledge: a functional MRI study of left inferior prefrontal cortex. Journal of Cognitive Neuroscience 1997;9:714-26.

[65] Wagner AD, Poldrack RA, Eldridge LL, Desmond JE, Glover GH, Gabrieli JDE. Material-specific lateralization of prefrontal activation during episodic encoding and retrieval. Neuroreport 1998;9:3711-7.

[66] Wagner AD, Schacter DL, Rotte M, Koutstaal W, Maril A, Dale AM, Rosen BR, et al. Building memories: remembering and forgetting of verbal experiences as predicted by brain activity. Science 1998;281:1188-91.

[67] Warburton E, Wise RJS, Price CJ, Weiller C, Hadar U, Ramsay S, et al. Noun and verb retrieval by normal subjects: studies with PET. Brain 1996;119:159-79.

[68] Warren C, Morton J. The effects of priming on picture recognition. British Journal of Psychology 1982;73:117-29.

[69] Warrington E, Taylor AM. Two categorical stages of object recognition. Perception 1978;7:695-705.

[70] Wiggs CL, Martin A. Properties and mechanisms of perceptual priming. Current Opinion in Neurobiology 1998;8:227-33. 